\title{
A New DG Planning Approach to Maximize Renewable - Based DG Penetration Level and Minimize Annual Loss
}

\author{
Soroush Najafi ${ }^{1}$, Miadreza Shafie-khah ${ }^{1}$, Neda Hajibandeh ${ }^{1}$, Gerardo J. Osório $^{1}$ and \\ João P. S. Catalão ${ }^{1,2,3}$ \\ ${ }^{1}$ C-MAST, University of Beira Interior, Covilhã 6201-001, Portugal \\ 2 INESC TEC and the Faculty of Engineering of the University of Porto, Porto 4200-465 \\ ${ }^{3}$ INESC-ID, Instituto Superior Técnico, University of Lisbon, Lisbon 1049-001, Portugal \\ soroush.najafi@ubi.pt; miadreza@ubi.pt; neda.hajibandeh@ubi.pt; gjosilva@gmail.com; \\ catalao@fe.up.pt
}

\begin{abstract}
Distributed Generation (DG) using renewable technologies is increasing due to their benefits including energy security and emission reduction. However, installing new DGs in distributed networks is limited due to network constraints such as feeder capacity and short circuit level, as well as higher investment costs. In this paper, network reconfiguration and reactive power planning are used to maximize DG penetration level and to minimize annual loss for DGs with biomass technologies. In order to model the problem uncertainties, 96 scenarios considering ten different network load levels are studied. A multi-objective method is applied for solving this optimization problem by using Pareto front. The numerical results indicate the positive impacts of the proposed approach on improving the network security.
\end{abstract}

Keywords: Annual loss, Biomass, DG penetration, Network reconfiguration, Pareto front, Reactive power planning, Renewable energy.

\section{Introduction}

Environmental concerns, such as global warming causes that renewable energy, e.g., biomass, play an important role in supplying energy [1]. The planning of distribution systems is a critical issue that the system planners faced with [2]. In one hand, appropriate placement of DGs in the distribution systems has an essential role in the enhancement of the system efficiency; consequently finding the optimum placement of DGs is critical [3]. On the other hand, a large number of researchers have focused on the reactive power planning in the distribution system, especially when it has have high-penetration of renewable resources DG [4].

In this sense, network reconfiguration is a method to change the topology of branches through altering the open and close statuses of sectionalizing and tie switches [3]. This can be carried out in different seasons of a year to reduce the total loss and increase the system reliability. 
For instance, as reported in [4], a DG planning was proposed for the coordination of reconfiguration of feeders and voltage controlling with the goal of maximizing the DG capacity at a specific bus.

In the current work, it is planned biomass DG for four different scenarios. In the first scenario, the annual loss is minimized without considering network penetration. The second scenario represents minimized annual loss considering network penetration. In the third scenario, network penetration is maximized, and finally, in the fourth scenario, a multi-objective problem is provided that minimizes the annual loss and maximizes the network penetration.

\section{Relationship to Smart Systems}

Distributed Generation (DG) of electricity using renewable technologies is increasing due to their benefits including energy security and emission reduction [5]. Smart grid can be defined as a contemporary structure of electricity systems to improve effectiveness, reliability, and safety, via integration of sustainable energies, as well as automated and intelligent controlling and advanced communications technologies [6], [7]. It affects entire parts of power systems, and consequently it needs a widespread data communication structure [8].

Importantly, smart grid allows modern network controlling schemes and provides operative integrated DGs in the demand side [9], [10]. Smart Grid will have a significant role to support high penetration level of DGs; nevertheless, the existing standard/code leading DGs' interconnection does not allow implementing various applications that can be advantageous for the system [11]. Novel approaches for utilizing the data in the supervisory controllers would be required, as well as an environment in which tools could be emerged in online stream event processing.

\section{PSO and MOPSO Algorithm}

The particle swarm optimization (PSO) applied in optimization techniques; it was inspired from the movement migration of population species that lives grouped [12]. The elementary philosophy behind PSO is that each individual from the swarm, hereafter called as particle, uses the information from the swarm, based on the knowledge, sharing, and historical information to find its survival means. Computationally, PSO process starts with a random swarm of particles delimited from delimited universe, where each particle, from the movement process, will be a momentary solution of the problem with tuned fitness rate. The aforementioned fitness is computed and optimized in each iteration. The movement rule, defined by a position and velocity, defines the direction, speed, and position of the swarm to reach the optimal and are updated in each iteration. In this process, the particles interact with each other and them they move from the delimited universe till reach the best solution (the optimal from the optimization process). Both, position and velocity are updated them from the experience from the past position and velocity and the best 
position found from the swarm. Computationally, the aforementioned description can be expressed:

$$
\begin{aligned}
& X_{p}^{\text {iter }+1}=X_{p}^{\text {iter }}+V_{p}^{\text {iter }} \\
& V_{P}^{\text {iter }+1}=V_{p}^{\text {iter }}+\operatorname{Rand}_{1}\left(X_{p, \text { best }}^{\text {iter }}-X_{p}^{\text {iter }}\right)+\operatorname{Rand}_{2}\left(X_{g, \text { best }}^{\text {iter }}-X_{p}^{\text {iter }}\right) \\
& \quad p=1,2, \ldots, N_{p}
\end{aligned}
$$

where $X_{p}^{\text {nter }}$ is the last position of a particle $p$ from iteration iter that may express the best result to the optimization problem. By other words, and specifically in DG optimization problem, $X_{p}^{\text {nter }}$ is a set of DG decision variables and $V_{p}^{\text {trer }}$ is the velocity from particle $p$ from iteration iter. Moreover, $X_{g}^{\text {itegest }}$ is the best global position $g$ found from the swarm in iteration iter. So, the movement direction of the swarm is dependent by the influence, respectively, from $V_{p}^{\text {iter }}$, and $X_{g}^{\text {itegest }}$ [13]. By other words, the swarm movement is strictly influenced by the inertia, memory and cooperation of the swarm, expressed by the three term of (2). However, for the multi-objective problem of DG optimization proposed in this work the original PSO strategy is modified. The original goal to find a unique solution is then turned in one new array of multiple solutions, based on Pareto strategy to solve the multi-objective DG problem [14], [15].

\section{Formulation}

The planning model aims the minimization from the yearly energy loss and maximizes network penetration in the distribution system for all possible loads. Time segments are represents in sets of $90 \mathrm{~h}$ (i.e., the number of days for each season). Moreover, in (3), $g$ represents as load states, and $P_{\text {loss }}(g)$ represents energy loss at $(g)$ load state. Furthermore, in (4), $C_{m}$ represents biomass capacity installed at each candid bus $(C B)$.

Minimize $\quad E_{\text {Loss }}=\sum_{g=1}^{N} P_{\text {loss }}(g) * 90$

Maximize Penetration $=\sum_{m} C_{m} \quad m \in C B$

Subject to:

Active and Reactive power balance:

$$
\begin{aligned}
& P_{D G B, i}-L P(g) * P_{D, i}=\sum_{j=1}^{n} V_{g, i} * V_{g, j} * Y_{i j} * \operatorname{Cos}\left(\theta_{i j}+\delta_{g, j}-\delta_{g, i}\right), \\
& i \in N B u s \\
& -L P(g) * Q_{D, i}=-\sum_{j=1}^{n} V_{g, i} * V_{g, j} * Y_{i j} * \operatorname{Sin}\left(\theta_{i j}+\delta_{g, j}-\delta_{g, i}\right), i \in N B u s
\end{aligned}
$$


In (5) and (6), $L P(g)$ represent load peak level that shown in Table 1. $V_{g, i}$ represents voltage magnitude at load level $g . P_{D, i}$ and $Q_{D, i}$ represent active and reactive demands, respectively.

Slack Bus Constraints:

$$
\begin{aligned}
& V_{g, 1}=0 \\
& \delta_{g, 1}=0
\end{aligned}
$$

Voltage Constraints at the Other Buses:

$$
V_{\text {min }} \leq V_{g, i} \leq V_{\text {max }}
$$

where $V_{\min }$ and $V_{\max }$ are 0.95 p.u and 1.05 p.u respectively.

Feeder Capacity Limits:

$$
0 \leq I_{g, i j} \leq I_{i j, \max }
$$

DG Penetration. To this end, it has 2 different type of DG penetration in DG planning. (11) represent DG penetration for candidate bus. This limitation depends on the land and protection level of the candidate bus. In this paper, it is assume this limitation at $1200 \mathrm{~kW}$. (12) limit installed DG capacity in the whole network. This limitation assumes to be $1200 \mathrm{~kW}$ too. (12) just consider in the 3rd scenario.

$$
\begin{aligned}
& P_{D G B, m} \leq 1200 \mathrm{~kW} \text { mò } C B \\
& \sum_{m} C_{m} \quad m \in C B
\end{aligned}
$$

\section{Numerical Results of the Model}

The PG\&E 69bus distribution test case [16] has been considered to implement the proposed model. In normal operation, disregarding DGs, the yearly energy loss is 199.5 MWh. The slack bus of the system is node 1. The candidate bus for installing $\mathrm{DG}$ is $\mathrm{CB}=\{20,46,49,50,53\}$. Total active and reactive demands are $3802 \mathrm{~kW}$ and $2695 \mathrm{kVAr}$, respectively. The 69bus case study is also presented in Fig. 1. Implemented DG in the network is biomass resources with the controllable output. The hourly demand is represented in Table 1 as a proportion of the yearly demand peak. The proposed planning problem is applied to 4 scenarios with different constraint and objective function. Outcomes are represented in Table 2. Based on the results, if it is added DG, penetration constraint to an optimization problem, annual loss would increase. 
Table 1. Load profile for different hours

\begin{tabular}{|c|c|c|c|c|}
\hline Time & Winter & Spring & Summer & Autum \\
\hline $12: 00-1: 00 \mathrm{am}$ & 0.4756 & 0.3970 & 0.64 & 0.3717 \\
\hline $1: 00-2: 00$ & 0.4473 & 0.3906 & 0.60 & 0.3558 \\
\hline $2: 00-3: 00$ & 0.4260 & 0.3780 & 0.58 & 0.3540 \\
\hline $3: 00-4: 00$ & 0.4189 & 0.3654 & 0.56 & 0.3422 \\
\hline $4: 00-5: 00$ & 0.4189 & 0.3717 & 0.56 & 0.3481 \\
\hline $5: 00-6: 00$ & 0.4260 & 0.4095 & 0.58 & 0.3835 \\
\hline $6: 00-7: 00$ & 0.5254 & 0.4536 & 0.64 & 0.4248 \\
\hline 7:00 - 8:00 & 0.6106 & 0.5355 & 0.76 & 0.5015 \\
\hline $8: 00-9: 00$ & 0.6745 & 0.5958 & 0.87 & 0.5605 \\
\hline 9:00 - 10:00 & 0.6816 & 0.6237 & 0.95 & 0.5841 \\
\hline $10: 00-11: 00$ & 0.6816 & 0.6300 & 0.99 & 0.5900 \\
\hline $11: 00-12: 00$ & 0.6745 & 0.6237 & 1.00 & 0.5841 \\
\hline $12: 00-1: 00 \mathrm{am}$ & 0.6745 & 0.5859 & 0.99 & 0.5487 \\
\hline 1:00 - 2:00 & 0.6745 & 0.5796 & 1.00 & 0.5428 \\
\hline $2: 00-3: 00$ & 0.6603 & 0.5670 & 1.00 & 0.5310 \\
\hline $3: 00-4: 00$ & 0.6674 & 0.5544 & 0.97 & 0.5192 \\
\hline $4: 00-5: 00$ & 0.7029 & 0.5670 & 0.96 & 0.5310 \\
\hline $5: 00-6: 00$ & 0.7100 & 0.5796 & 0.96 & 0.5428 \\
\hline $6: 00-7: 00$ & 0.7100 & 0.6048 & 0.93 & 0.5664 \\
\hline $7: 00-8: 00$ & 0.6816 & 0.6174 & 0.92 & 0.5782 \\
\hline $8: 00-9: 00$ & 0.6461 & 0.6048 & 0.92 & 0.5664 \\
\hline $9: 00-10: 00$ & 0.5893 & 0.5670 & 0.93 & 0.5310 \\
\hline $10: 00-11: 00$ & 0.5183 & 0.5040 & 0.87 & 0.4720 \\
\hline $11: 00-12: 00 \mathrm{am}$ & 0.4473 & 0.4410 & 0.72 & 0.4130 \\
\hline
\end{tabular}

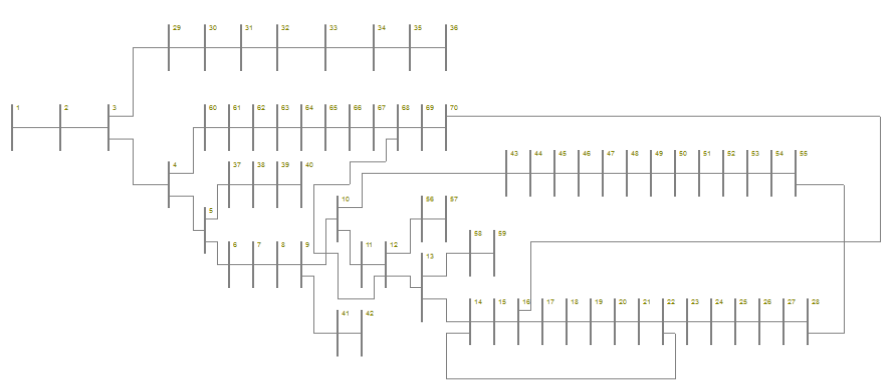

Fig. 1. 69bus PG\&E Single Diagram

Another effect of adding this constraint is that it is not possible to control voltage bus within 0.95 to 1.05 per-units (p.u). In this scenario, the buses voltage is varied 
from 0.9 to 1.1. In the third scenario, it is maximized DG penetration without violation any constraints. In this case voltage limit is $0.95<\mathrm{V}<1.05$. In the fourth scenario is planning biomass DG with two objective functions. Pareto optimal front found by the algorithm is shown in Fig 2. In this scenario voltage limit will be in 0.95 to 1.05 p.u.

TOPSIS algorithm is implemented to find the best decision for the decision maker. The theory of this algorithm is represented in [17]. The answers are represented in Table 3. It is used three different set of weight factor for objectives. Weight factors are $\{(0.2,0.8),(0.5,0.5),(0.8,0.2)\}$. The result is shown in Table 3 .

Table 2. DG Planning results for different scenarios

\begin{tabular}{l|l|l|l}
\hline \multirow{2}{*}{$\begin{array}{l}\text { Candidate } \\
\text { Bus }\end{array}$} & $\begin{array}{l}\text { Annual Loss } \\
\text { minimization } \\
\text { TA(With Consider DG } \\
\text { Penetration) }\end{array}$ & $\begin{array}{l}\text { Annual } \\
\text { minimization } \\
\text { (Without Consider DG } \\
\text { Penetration) }\end{array}$ & $\begin{array}{l}\text { DG } \\
\text { Penetration } \\
\text { Maximization }\end{array}$ \\
\hline 20 & 560 & 0 & 1600 \\
\hline 46 & 90 & 0 & 0 \\
\hline 49 & 0 & 0 & 3020 \\
\hline 50 & 480 & 0 & 1160 \\
\hline 53 & 1235 & 1200 & 0 \\
\hline $\begin{array}{l}\text { Annual Loss } \\
\text { KWh) }\end{array}$ & 2365 & 1200 & 5760 \\
\hline $\begin{array}{l}\text { Total } \\
\text { Installed } \\
\text { Capacity }\end{array}$ & 252120 & 313240 & 388510 \\
\hline
\end{tabular}

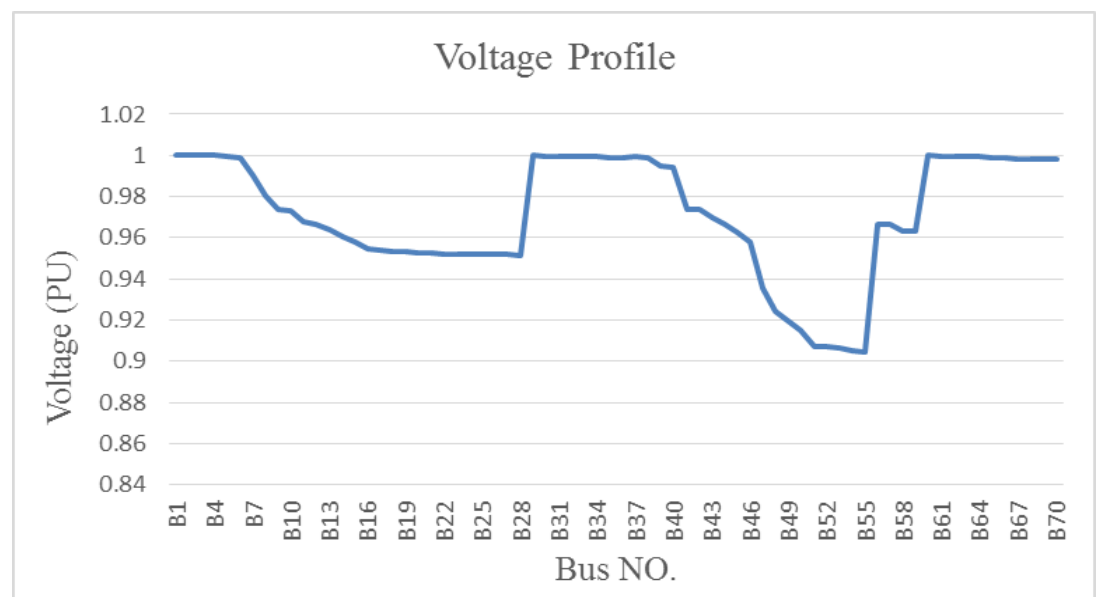

Fig. 2. Impact of different types of TOU program on the generation of Genco 1 without the presence of wind farm. 


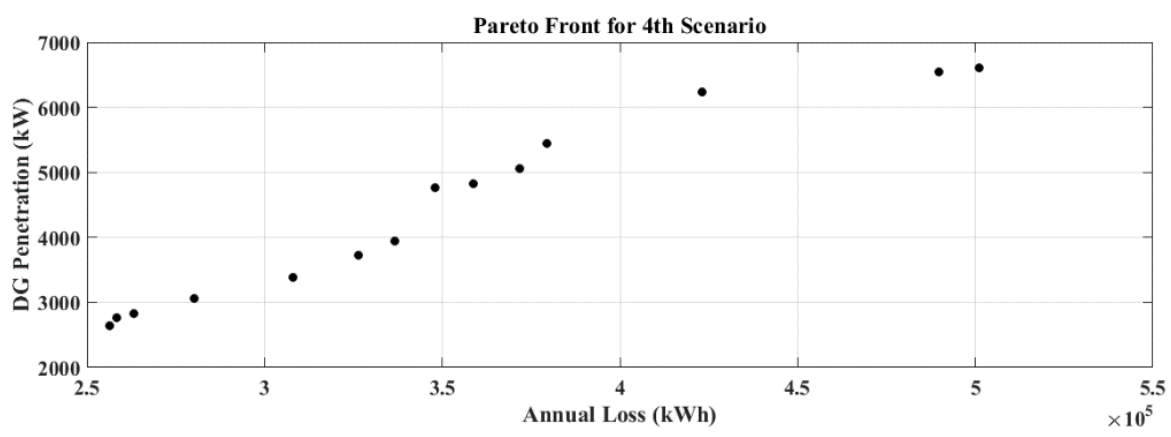

Fig. 3. Pareto Front for $4^{\text {th }}$ Scenarios.

Table 3. PLANNER Best Decision based on different weight factors

\begin{tabular}{l|l|l}
\hline Weight Factor & Annual Loss $(\mathrm{kWh})$ & DG Penetration \\
\hline$(0.2,0.8)$ & 258310 & 2770 \\
\hline$(0.5,0.5)$ & 423140 & 6239 \\
\hline$(0.8,0.2)$ & 423140 & 6239 \\
\hline
\end{tabular}

\section{Conclusions}

In this work a multi-objective model was presented for the distributed generation planning and solved the formulated problem with PSO algorithm. DGs were biomass with controllable output, although the proposed strategy could just tackle several types of uncertainty from wind or solar energy, for instance. Annual loss minimization and DG penetration maximization were considered in this paper. After determining the Pareto front, it was used TOPSIS method to choose the best decision for the decision maker. The numerical results showed that considering only the DG penetration constraint increased the annual loss. In addition, by adding this constraint, the voltage bus could not be constrained between desired amounts. However, the proposed approach considering both DG penetration constraint and annual loss could significantly improve the effectiveness of the DG planning.

Acknowledgment. This work was supported by FEDER funds through COMPETE 2020 and by Portuguese funds through FCT, under Projects SAICT-PAC/0004/2015 POCI-01-0145-FEDER-016434, POCI-01-0145-FEDER-006961, UID/EEA/50014/2013, UID/CEC/50021/2013, and UID/EMS/00151/2013. Also, the research leading to these results has received funding from the EU Seventh Framework Programme FP7/2007-2013 under grant agreement no. 309048. 


\section{References}

1. Y.M. Atwa, E.F.El Saadany: Probabilistic approach for optimal allocation of wind based distributed generation in distribution systems. IET Renew. Power Gener. 5, 79-88 (2011).

2. S.A. Arefifar, Y.I.M. Abdel-Rady: Probabilistic optimal reactive power planning in distribution systems with renewable resources in grid-connected and islanded modes. IEEE Trans. Ind. Elect. 61(11), 5830-5839 (2014).

3. Y.M. Atwa, E.F. El Sadaany, M.M.A. Salama, R. Seethapathy: Optimal renewable resource mix for distribution system energy loss minimization. IEEE Trans. Power Syst. 25(1), 360$370(2010)$.

4. S.-Y. Su, C.-N. Lu, R.-F. Chang, G. Gutiérrez-Alcaraz. Distributed generation interconnection planning: a wind power case study. IEEE Trans. Smart Grid 2(1), 181-189 (2011).

5. V. C. Gungor, B. Lu, G. P. Hancke. Opportunities and challenges of wireless sensor networks in smart grid. IEEE Trans. Ind. Electron., 57(10), 3557-3564 (2010).

6. C. Cecati, C. Citro, P. Siano. Smart operation of wind turbines and diesel generators according to economic criteria. IEEE Trans. Ind. Electron., 58(10), 4514-4525 (2011).

7. H. Arasteh et al. IoT-based smart cities: A survey. In Proc. 2016 IEEE 16th Int. Conf. Environment and Electrical Engineering (EEEIC), Florence, (2016), 1-6.

8. F. Kamyab, M. H. Amini, S. Sheykhha, M. Hasanpour, and M.M. Jalali. Demand response program in smart grid using supply function bidding mechanism. IEEE Trans. Smart Grid, 7(2), 1277-1284 (2016).

9. P. Palensky, D. Dietrich. Demand side management: Demand response intelligent energy systems and smart loads. IEEE Trans. Ind. Inform., 7(3), 381-388 (2011).

10. V. Calderaro, C. Hadjicostis, A. Piccolo, P. Siano. Failure identification in smart grids based on Petri net modeling. IEEE Trans. Ind. Electron., 58(10), 4613-4623 (2011).

11. S. Bahrami, A. Sheikhi. From demand response in smart grid toward integrated demand response in smart energy hub. IEEE Trans. Smart Grid, 7(2), 650-658 (2016).

12. M. Couceiro, P. Ghamisi. Fractional order Darwinian particle swarm optimization

applications and evaluation of an evolutionary algorithm. Springer Briefs in Appl. Sciences Tech., 1 Ed., p.75 (2016).

13. A. Soroudi, M Afrasiab. Binary PSO-based dynamic multi-objective model for distributed generation planning under uncertainty. IET Renew. Power Gener., 6(2), 67-78 (2012)

14. S. Devi, A. K. Agade, S. Dehuri. Comparison of various approaches in multi-objective particle swarm optimization (MOPSO): Empirical study. Studies in Computational Intelligence, 592, 75-103 (2015).

15. S.F. Santos, et al. Novel multi-stage stochastic DG investment planning with recourse. IEEE Trans. Sust. Energy, 8(1), 164-178 (2016).

16. M.E Baran, F.F. Wu. Optimal capacitor placement on radial distribution systems. IEEE Trans. Power Deliv., 4(1), 725-734 (1989).

17. A. M. Eldurssi, R. M. O'Connell. A fast nondominated sorting guided genetic algorithm for multi-objective power distribution system reconfiguration problem. IEEE Trans. Power Syst., 30(2), 593-601 (2015). 